\title{
A Hybrid NSGA-II Algorithm for Multiobjective Quadratic Assignment Problems
}

\author{
Z. KAMisli OzTURK ${ }^{a, *}$ And M. Uluel ${ }^{b}$ \\ ${ }^{a}$ Anadolu University, Industrial Engineering Department, Eskisehir, Turkey \\ ${ }^{b}$ TUSAS Engine Industry, Eskisehir, Turkey
}

\begin{abstract}
In this study, we propose a novel hybrid multiobjective evolutionary algorithm for solving multiobjective quadratic assignment problems. During the last decade, the researchers gave increasing attention to the multiobjective structure of quadratic assignment problems and developed and/or used several multi objective metaheuristics. The nondominated sorting genetic algorithm (NSGA-II) has been shown to solve various multiobjective problems much better than other recently-proposed constraint handling approaches. Besides, the effectiveness of conic scalarization method was also proven for solution of multiobjective problems, that have non-linear structure. Here, a hybrid multiobjective evolutionary algorithm (cNSGA-II) featured with NSGA-II and conic scalarization's Pareto solutions is developed to obtain as much Pareto points, as possible. To test the performance of the algorithm we have selected the test problems from the literature and compared the performances by well-known diameter metric. It has been shown that cNSGA-II is effective in solving multiobjective quadratic assignment problems.
\end{abstract}

DOI: 10.12693/APhysPolA.132.959

PACS/topics: Hybrid NSGA-II, Multiobjective Quadratic Assignment, Conic Scalarization

\section{Introduction}

Quadratic assignment problems (QAP) were firstly defined by Koopmans and Beckmann [1]. QAP have a number $n$ of locations and facilities. There is a flow between these locations and facilities. Each facility is assigned to a location and also each location is assigned to a facility. For this problem, the objective is minimization of total assignment costs. In real life, these problems are encountered in hospital layouts, in design of keyboards and in circuit analysis. Combinatorial data analysis problems can also be modeled as a QAP. While the constraints of QAP are $0-1$ assignment constraints, the objective function is nonlinear, because of the quadratic structure. Based on these two reasons, QAP is classified as NP-Hard, and it is almost impossible to find optimal solutions if the number of instances is greater than 20 [2]. Thus, besides the exact solution methods to solve this kind of problems, metaheuristic algorithms like genetic algorithms, tabu search and neural networks $[3,4]$ have been proposed.

Based on the multi objective structure of real life problems [5-7], multiobjective quadratic assignment problems (mQAP) were suggested by Knowles and Corne [2]. The difference of mQAP from the QAP is that it has multiple flow matrices $(m>2)$. mQAP is also a NP-Hard problem like QAP. In addition, more than one nonlinear objective functions have to be considered. The objective of mQAP is [2]:

$$
\min C(\pi)=\left\{C^{1}(\pi), C^{2}(\pi), \ldots, C^{k}(\pi)\right\},
$$

where $C^{k}(\pi)=\sum_{i=1}^{n} \sum_{j=1}^{n} a_{i j} b_{\pi_{i} \pi_{j}}^{k}, k \in \overline{1, m}, n$ is the number of facilities/locations, $m$ is the number of

*corresponding author; e-mail: zkamisli@anadolu.edu.tr different flow matrices, $a_{i j}$ is the distance between locations $i$ and $j, b_{i j}^{k}$ is the $k^{t h}$ flow from facility $i$ to facility $j$, $\pi_{i}$ is the location of $i^{t h}$ facility in permutation $\pi \in P(n)$, and $P(n)$ is the set of all permutations.

Knowles and Corne have again studied mQAP in 2003 [8]. After this, different researchers have studied this subject, using various solution approaches. These studies were about heuristic and metaheuristic algorithms. Garrett and Dasgupta [9, 10] provided a comparison of a number of strategies for the construction of multiobjective memetic algorithms with some local search strategies for the mQAP. Paquete and Stützle [11] also tried to find Pareto solutions with some local search algorithms. Their proposed local search algorithms gave good quality solutions in reasonable times. $\mathrm{Li}$ and Silva [12] proposed a GRASP algorithm for mQAP. Özkale and Fı̆̆lalı [13] presented another study considering a metaheuristic approach (ant colonies optimization) for mQAP. Almeida et al. [14] have published one of the latest studies that proposed a hybrid algorithm of transgenetic algorithm and NSGA-II. Aside from the main studies in the literature, Zhao et al. [15] presented a fuzzy solution approach for mQAP, called fuzzy particle swarm algorithm. Ammar [16] also considered portfolio optimization problem as a fuzy mQAP. Ammar [17] continued the fuzzy mQAP studies with relative scalar quadratic multiobjective programming with fuzzy coefficients.

According to the literature review it is obvious that to solve mQAP, efficient metaheuristic and/or hybrid metaheuristic algorithms are needed. In this study, in order to propose an efficient solution approach to mQAP, firstly the conic scalarization method and multiobjective evolutionary algorithm of NSGA-II were used to find Pareto solutions. Then, by considering the strengths of both methods we propose a hybrid solution approach. The novel hybrid approach and the numerical results are 
presented in Section 2 and the conclusions are given in in Section 3. Test problems from the literature were used to compare the efficiency of the proposed solution approach.

\section{A hybrid NSGA-II algorithm for multiobjective QAP}

Based on the multiobjective structure of mQAP, we should concentrate on the Pareto solutions. There are some scalarization methods to find Pareto solutions. Effectiveness of conic scalarization method (CS) (ability to find Pareto solutions) is proven for solution of multiobjective problems, especially the ones that have non-linear structure [18]. Besides the scalarization methods, multiobjective evolutionary algorithms (MOEA) are also used to find Pareto solutions.

Non-dominated sorting genetic algorithm-II (NSGA-II) is one of these MOEA, and its ability to find Pareto solutions is also proven to be efficient [19, 20].

In their study, Kasimbeyli et al. [18] presented main features of the conic scalarization method (CS) in multiobjective optimization problems. It is proven that CS method is guaranteed to generate proper efficient solutions and does not require any kind of convexity or boundness conditions. The idea of the CS method is very simple: choose preference parameters which consist of a weight vector $w \in C^{a \#}$ and a reference point $a \in R^{n}$, determine an augmentation parameter $\alpha \in R_{+}$, such that $(w \alpha) \in C^{a *}\left(\right.$ or $(w \alpha) \in C^{a \circ}$ or $\left.(w \alpha) \in C^{a \#}\right)$, where for a convenience the $l_{1}$-norm is used, and solve the scalar optimization problem $C S(w, \alpha, a)$ in Eq. (2).

The set of optimal solutions of this scalar problem will be denoted by $\operatorname{Sol}(C S(w, \alpha, a))$. Reference point $a=\left(a_{1}, \ldots, a_{n}\right)$ may be identified by a decision maker in cases when she/he desires to calculate minimal elements that are close to some point. The CS method does not impose any restrictions on the ways for determining reference points. The reference point can be chosen arbitrarily.

$$
\begin{aligned}
& \min _{x \in X} \sum_{i=1}^{n} w_{i}\left(f_{i}(x)-a_{i}\right) \\
& \quad+\alpha \sum_{i=1}^{n}\left|f_{i}(x)-a_{i}\right| C S(w, \alpha, a) .
\end{aligned}
$$

In the first step of the NSGA-II algorithm, random population $P_{t}$ with size $N$ is generated based on the problem's range and constraints. Then, all objective function values are evaluated. Nondominated and crowding distances are used to sort these solutions. After that, with the selection, crossover and mutation operators, the offspring population $Q_{t}$ with size $N$ is created. The solutions of offspring population are then evaluated. Then, the population $R_{t}$ is formed by the populations $P_{t}$ and $Q_{t}$. The population size of $R_{t}$ is $2 N$.

The solutions of $R_{t}$ are sorted in different nondominated front. In the new population $P_{t+1}$, the best solutions are added to fulfill the new generation, if the number of these solutions is less than the population size. These best solutions are the solutions in the best front and with the best value of the crowding distance.

To compare solutions of these two methods, the instances which were presented by Knowles and Corne [8] were used in this study. These instances have 10 locations and 2 objectives, 20 locations and 2 objectives, 30 locations and 2 objectives, 30 locations and 3 objectives. The instances were solved with CS via GAMS software (Version 23.3), and with NSGA-II via VBA. Unlike in single-objective optimization, we need to use some metrics to measure the performance of multiobjective solution algorithm.

In a multiobjective optimization there are two goals [20]: 1) convergence to the Pareto optimal set and 2) maintenance of diversity in solutions of the Pareto optimal set. In this study, the diameter metric, which is given in Eq. (3) is used. Let $\operatorname{dist}(\pi, \mu)$ is the distance between solution $\pi$ and $\mu$,

$$
\operatorname{dmm}(P)=\sum_{\pi \in P} \sum_{\mu \in P} \operatorname{dist}(\pi, \mu)|P|^{2} .
$$

The diameter values obtained for CS method and NSGA-II are given in Table I. For some instances CS gave better diameter values, and for the other, NSGA-II. In Fig. 1, the Pareto solutions obtained by $\mathrm{CS}$ and NSGA-II are given for the instances KC10-2fl-1rl and KC10-2fl-1uni. It is clear that more Pareto solutions were obtained by CS. However, for the large scale instances CS gave better diameter values and gave almost all Pareto solutions. The solution time is not reasonable, because solution time of nonlinear constrained optimization problems depends on the number of constraints, decision variables and conditioning of decision variables space [22].

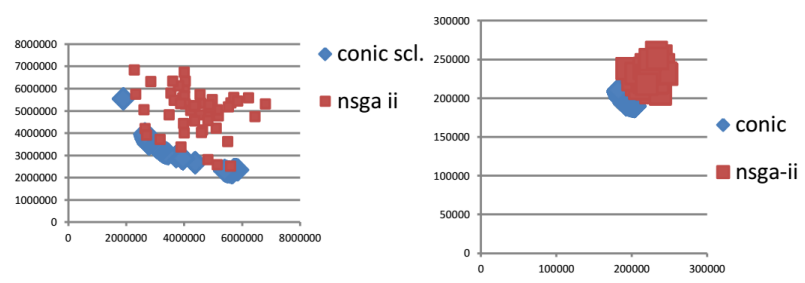

Fig. 1. Pareto solutions obtained by CS and NSGA-II.

NSGA-II is also good at finding Pareto solutions and is faster than NSGA-II for the large scale problems. Based on these facts, a novel hybrid multiobjective evolutionary algorithm, based on conic scalarization and NSGA-II is proposed.

The main structure of our proposed hybrid NSGA-II (cNSGA-II) algorithm is same as that of NSGA-II. The novel difference is that $20 \%$ of the initial population is taken from the Pareto solutions, obtained by conic scalarization. The procedure of cNSGA-II is given in Fig. 2.

All diameter metric values of NSGA-II, CS and the cNSGA-II are given in Table I. The performance of the hybrid algorithm is better in 9 of 17 instances. 
TABLE I

Diameter values obtained by NSGA-II, CS and cNSGA-II.

\begin{tabular}{|c|c|c|c|c|c|c|c|}
\hline Problem & NSGA-II & $\mathrm{CS}$ & cNSGA-II & Problem & NSGA-II & CS & cNSGA-II \\
\hline KC10-2fl-1rl & 370.318 & 432.882 & 449.990 & KC20-2fl-1uni & 10.465 & 30.590 & 58.935 \\
\hline KC10-2fl-1uni & 4.416 & 6.378 & 6.451 & KC20-2fl-2rl & 776.318 & 760.189 & 764.382 \\
\hline KC10-2fl-2rl & 325.855 & 155.762 & 312.896 & KC20-2fl-2uni & 9.295 & 5.233 & 80.814 \\
\hline KC10-2fl-2uni & 4.458 & 782 & 20.980 & KC20-2fl-3rl & 718.149 & 1.176 .523 & 890.217 \\
\hline KC10-2fl-3rl & 367.312 & 401.294 & 417.904 & KC20-2fl-3uni & 23.176 & 54.994 & 63.379 \\
\hline KC10-2fl-3uni & 4.842 & 16.860 & 16.013 & KC20-2fl-4rl & 4.134.239 & 2.551 .831 & 4.664 .329 \\
\hline KC10-2fl-4rl & 389.446 & 380.342 & 354.249 & KC20-2fl-5rl & 4.652 .019 & 5.551 .017 & 4.450 .468 \\
\hline KC10-2fl-5rl & 1.610 .559 & 515.146 & 1.672 .450 & KC30-2fl-1rl & 1.183 .783 & 1.973 .953 & 1.337 .680 \\
\hline $\mathrm{KC} 20-2 \mathrm{fl}-1 \mathrm{rl}$ & 956.204 & 1.639 .296 & 1.374 .347 & & & & \\
\hline
\end{tabular}

TABLE II

Diameter values obtained by cNSGA-II, MOMGA-II, MOMGA-IIa and Knowles and Corne.

\begin{tabular}{|c|c|c|c|c|c|}
\hline Problem & $\begin{array}{c}\text { Knowles and Corne } \\
\text { results [8] }\end{array}$ & MOMGA-II [20] & MOMGA-IIa [21] & Conic scalarization & cNSGA-II \\
\hline KC10-2fl-1rl & 7.000 & - & - & 432.882 & 449.990 \\
\hline KC10-2fl-1uni & 7.000 & - & - & 6.378 & 6.451 \\
\hline KC10-2fl-3uni & 8.000 & - & - & 16.860 & 16.013 \\
\hline KC20-2fl-1uni & 15.000 & 11.400 & 13.700 & 30.590 & 58.935 \\
\hline KC20-2fl-2uni & 14.000 & 7.200 & 3.670 & 5.233 & 80.814 \\
\hline KC20-2fl-3uni & 16.000 & 12.300 & 15.500 & 54.994 & 63.379 \\
\hline
\end{tabular}

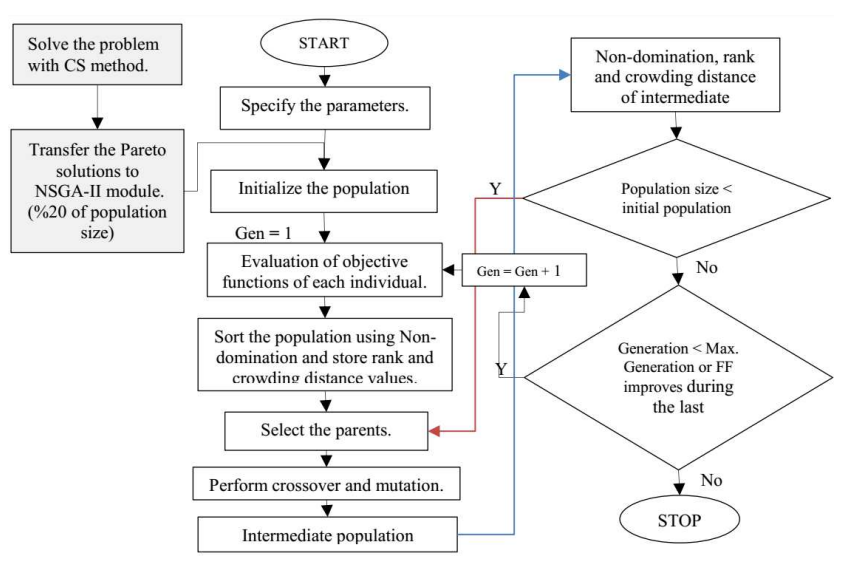

Fig. 2. Procedure of the novel cNSGA-II algorithm.

Obtained results were also compared with the results of the other methods in the literature (Table II). It is also clear that cNSGA-II gives better results than the results published in literature.

\section{Conclusions}

In this study, mQAP which is a relatively new subject in the literature, is considered. The contribution to the literature of this study is two fold. The exact solution methodology to mQAP with conic scalarization method is firstly studied. As the second contribution, based on the strengths of CS and NSGA-II, a novel hybrid NSGA-II (cNSGA-II) is proposed.

Instances presented by Knowles and Corne [8] were used in this study and were first solved with CS and NSGA-II. For different problems, we see that both of these methods give better solutions in particular cases. However neither of these methods dominates the other. Results obtained with proposed hybrid algorithm (cNSGA-II) were compared with the ones obtained with CS, NSGA-II and with other three methods from the literature. It has been shown that hybrid algorithm gives better solutions than the others.

\section{Acknowledgments}

This study is supported by Anadolu University Scientific Research Projects Committee (AUBAP-1601F041).

\section{References}

[1] T.C. Koopman, B. Beckmann, Econometrica, 531 (1957).

[2] J. Knowles, D. Corne, in: Soft Computing Systems: Design, Management and Applications, Ed. A. Abraham, IOS Press, Amsterdam 2002, p. 271.

[3] İ. H. Karahan, R. Özdemir, Acta Phys. Pol. A $\mathbf{1 2 8}$ B-427 (2015).

[4] E. Kanca, F. Çavdar, M.M. Erşen, Acta Phys. Pol. A 130, 365 (2016) 
[5] A.T. Özturhan, Acta Phys. Pol. A 130, 14 (2016).

[6] P. Stefanov, A. Savic, G. Dobric, Acta Phys. Pol. A 128, B-138 (2015).

[7] A. Recioui, Acta Phys. Pol. A 128, B-7 (2015).

[8] J. Knowles, D. Corne, Instance generator and test suites for the mQAP, Evolutionary Multi-Criterion $O p$ timization (EMO), in: Second International Conference, Portugal 2003.

[9] D. Garrett, D. Dasgupta, in: IEEE Congress on Evolutionary Computation, Vancouver 2006, p. 1710.

[10] D. Garrett, D. Dasgupta, in: IEEE Symposium on Computational Intelligence in Multi Criteria Decision Making, Nashville 2009, p. 80.

[11] L. Paquete, T. Stützle, Eur. J. Operat. Res. 169, 943 (2006).

[12] H. Li, D. Silva, LNCS 5467, 481 (2009).

[13] C. Özkale, A. Fiğlalı, Appl. Math. Model. 37, 7822 (2013).

[14] C.P. Almeida, E.F. Goldberg, M.R. Delgado, in: Brazilian Conf. Intelligent Systems, IEEE, 2014, p. 312.

[15] M. Zhao, A. Abraham, C. Grosan, H. Liu, in: 2nd Asia Int. Conf. Modelling \& Simulation, IEEE, 2008, p. 516.
[16] E.E. Ammar, Inform. Sci. 178, 468 (2008).

[17] E.E. Ammar, Eur. J. Operat. Res. 193, 329 (2009).

[18] R. Kasimbeyli, Z.K. Öztürk, N. Kasimbeyli, G.D. Yalçin, B. Içmen, Conic scalarization method in multiobjective optimization and relations with other scalarization methods, in: Modelling, Computation and Optimization in Information Systems and Management Sciences, Proceedings of MCO 2015, Eds. L.T.H. An, P.D. Tao, N.N. Thanh, vol. 359, part V, Springer, 2015.

[19] E. Zitzler, M. Laumanns, L. Thiele, SPEA2: Improving the strength Pareto evolutionary algorithm, TIKReport 103, 2001.

[20] K. Deb, A. Pratap, S. Agarwal, T. Meyarivan, IEEE Trans. Evolut. Comput. 6, 182 (2002).

[21] R.O. Day, G.B. Lamont, Multiobjective quadratic assignment problem solved by an explicit building block search algorithm MOMGA-IIa, LNCS 3448, SpringerVerlag, Berlin Heidelberg, p. 91.

[22] M.J. Pazdanowski, Acta Phys. Pol. A 128, B-213 (2015). 